Auteurs invités

\title{
UNE APPROCHE TYPOLOGIQUE DE L'ENTREPRENEURIAT DE NÉCESSITÉ
}

Christel Tessier-Dargent, Alain Fayolle

ARIMHE | « RIMHE : Revue Interdisciplinaire Management, Homme \& Entreprise » 2016/3 n²2 | pages 74 à 92

ISSN 2259-2490

Article disponible en ligne à l'adresse :

http://www.cairn.info/revue-rimhe-2016-3-page-74.htm

\section{Pour citer cet article :}

Christel Tessier-Dargent, Alain Fayolle« Une approche typologique de l'entrepreneuriat de nécessité », RIMHE : Revue Interdisciplinaire Management, Homme \& Entreprise 2016/3 (n²2), p. 74-92.

DOI 10.3917/rimhe.022.0074

Distribution électronique Cairn.info pour ARIMHE.

(C) ARIMHE. Tous droits réservés pour tous pays.

La reproduction ou représentation de cet article, notamment par photocopie, n'est autorisée que dans les limites des conditions générales d'utilisation du site ou, le cas échéant, des conditions générales de la licence souscrite par votre établissement. Toute autre reproduction ou représentation, en tout ou partie, sous quelque forme et de quelque manière que ce soit, est interdite sauf accord préalable et écrit de l'éditeur, en dehors des cas prévus par la législation en vigueur en France. Il est précisé que son stockage dans une base de données est également interdit. 


\section{Auteurs invités}

\section{Une approche typologique de l'entrepreneuriat de nécessité}

\section{Christel TESSIER-DARGENT ${ }^{14}$ Alain FAYOLLE ${ }^{15}$}

Les entrepreneurs par nécessité sont considérés comme « des individus poussés à la création d'entreprise car ils ne perçoivent pas de meilleure alternative d'emploi » (Cowling, Bygrave, 2003, p.544). Ils sont opposés, dans une dichotomie communément acceptée, aux entrepreneurs par opportunité, à savoir ceux qui poursuivent une opportunité d'affaires qu'ils ont identifiée ou créée.

Les entrepreneurs par nécessité font l'objet d'un nombre croissant de travaux de recherche depuis le début des années 2000, lesquels tendent à orienter des politiques publiques spécifiques à l'égard de cette catégorie d'entrepreneurs, tant dans les pays dits en développement que dans les économies développées. L'étude de la littérature internationale indique qu'avant 2000, dix articles ont été publiés sur le thème de l'entrepreneuriat de nécessité dans des revues de gestion classées A et B par le HCERES (Haut Conseil de l'Evaluation de la Recherche et de l'Enseignement Supérieur), contre quarante-cinq articles publiés entre 2000 et 2014. La dichotomie entre entrepreneurs par nécessité et par opportunité est issue des travaux de Shapero (1975) sur les motivations positives et négatives à créer. Celle-ci démontre que la décision d'entreprendre est corrélée au contexte dans lequel l'entrepreneur se situe. Elle observe que la plupart des entrepreneurs ont subi un «accident » dans leur vie personnelle ou professionnelle, qu'il nomme « déplacement ": licenciement, insatisfaction dans le travail, divorce, deuil, maladie, tout événement qui peut conduire un individu à entreprendre. Cette notion de déplacement, issue de l'approche psychanalytique, ouvre la porte à une vision non plus uniquement fonctionnelle, mais contingente, de l'entrepreneur. Ces circonstances peuvent être des éléments positifs (push) ou négatifs (pull). Cette dichotomie a été proposée par le consortium GEM ${ }^{16}$ (Reynolds et al., 2001) pour expliquer le phénomène d'un entrepreneuriat de survie

\footnotetext{
${ }^{14}$ Docteur en sciences de gestion, Université UPMF Grenoble-Alpes, CERAG/CNRS (UMR 5820) christel.tessierdarg@free.fr

${ }^{15}$ Professeur, EM Lyon Business School, CERAG/CNRS (UMR 5820) - fayolle@em-lyon.com

${ }^{16}$ Le «Global Entrepreneurship Monitor» (http://gemconsortium.org) est un consortium de recherche académique à but non lucratif dont l'objectif est de fournir des données rigoureuses sur l'activité entrepreneuriale mondiale. Il s'agit du plus important projet de ce type : initiées en 1999 avec 10 pays, les recherches ont été conduites en 2015 sur plus de 100 pays ; plus de 200000 entrepreneurs et experts ont été interrogés.
}

RIMHE - Revue Interdisciplinaire Management, Homme \& Entreprise

n²2 - Eté 2016 


\section{Auteurs invités : Une approche typologique de l'entrepreneuriat de nécessité Christel TESSIER-DARGENT, Alain FAYOLLE}

dans les pays en développement, puis a été étendue aux pays développés, compte-tenu de la globalisation du problème du chômage. GEM utilise ce terme pour décrire une création d'entreprise par défaut, sans projet préalable, répondant à une nécessité purement économique, d'obtenir un revenu, faute d'alternative professionnelle perçue. Ainsi, en lien avec le terrain, la recherche a ouvert un champ d'analyse critique du mythe schumpétérien de l'entrepreneur, décrivant habituellement une logique entrepreneuriale individuelle et victorieuse, pour passer à la description d'un processus social émergent et fortement contextualisé.

Deux approches divergentes orientent cet intérêt accru pour l'entrepreneuriat de nécessité. D'une part, dans la lignée de Schumpeter, Gartner et du GEM, des chercheurs voient dans l'entrepreneuriat de nécessité un entrepreneuriat de « deuxième catégorie » avec, en arrière-pensée, l'objectif de mieux diriger les investissements publics ou privés, non vers ces individus voués à l'échec, mais vers les gazelles innovantes, créatrices d'emploi et vecteurs de croissance (Girard, 2007 ; Shane, 2009 ; Schoar, 2009). S'expriment ici de fortes réticences vis-à-vis de l'entrepreneuriat de nécessité : son intérêt pour le développement économique est souvent perçu comme inférieur à celui de l'entrepreneuriat par opportunité, avec des taux de défaillance plus élevés, des tailles d'entreprises inférieures, une contribution économique, un développement des structures et une satisfaction des entrepreneurs moindres (Acs et Varga., 2005 ; Block et Wagner, 2010 ; Van der Zwan et Hessels, 2013). Thurik, Carree, Van Steel et Audretsch (2008) concluent d'ailleurs à la prédominance de l'effet Schumpeter sur l'effet « refuge » : le chômage peut certes susciter la création de nouvelles entreprises, mais c'est principalement la dynamique économique qui favorise la création d'entreprises, celles-ci réduisant le chômage en créant des emplois en retour. D'autre part, à l'inverse, pour répondre aux enjeux des crises économiques successives, les politiques publiques de nombreux Etats et les instances mondiales encouragent l'entrepreneuriat, sous toutes ses formes, comme outil de résorption du chômage et de réinsertion, invitant chaque individu «désavantagé » à prendre ses responsabilités et son destin en main ${ }^{17}$. La Commission Européenne souligne ainsi son objectif de promouvoir l'entrepreneuriat auprès de publics spécifiques, à savoir les femmes, les seniors, les migrants et les personnes au chômage, comme "une précieuse opportunité d'inclusion sociale ${ }^{18}$. Les critiques de la communauté de recherche à l'égard d'une promotion de cette forme d'entrepreneuriat de nécessité sont également

\footnotetext{
${ }^{17}$ Discours d'Angel Gurría, Secrétaire général de l'OCDE, G20 Young Entrepreneurs Summit, (2011): "L'entreprenariat est à n'en pas douter un formidable levier de politique publique pour promouvoir une reprise riche en emploi, solidaire, soucieuse de l'environnement et qui contribue à réduire le chômage des jeunes » - http://www.oecd.org/fr/apropos/secretairegeneral/g20youngentrepreneurssummit.html

${ }^{18}$ Article du site de la Commission Européenne (2013) : «Unleashing Europe's entrepreneurial potential to bring back growth » - http://www.ec.europa.eu/growth/tools-databases/newsroom
}

RIMHE - Revue Interdisciplinaire Management, Homme \& Entreprise

n²2 - Eté 2016 


\section{Auteurs invités: Une approche typologique de l'entrepreneuriat de nécessité Christel TESSIER-DARGENT, Alain FAYOLLE}

vives et nombreuses (Blackburn et Ram, 2006 ; Bergmann et Sternberg, 2007 ; Fayolle et Nakara, 2012 ; Levratto et Serverin, 2012 ; Abdelnour et Lambert, 2014). Ceux qui se lancent ont épuisé toutes les possibilités de trouver un emploi pour acquérir des revenus. Ils subissent plus qu'ils ne le désirent, l'entrepreneuriat, et les conséquences humaines, sociales et économiques de ce phénomène sont globalement négatives. Cependant, les travaux de Giacomin, Guyot, Janssen et Lohest (2007) démontrent qu'il ne faut pas systématiquement associer chômage et entrepreneuriat de nécessité. Ainsi, comme le rappelle Couteret (2010, p.3) : «Tout chômeur-créateur ne peut pas être qualifié d'entrepreneur contraint. Il existe des entrepreneurs dont les motivations relèvent à la fois de l'envie et de la nécessité ». La littérature souligne, à l'évidence, la complexité du concept d'entrepreneur par nécessité et l'hétérogénéité du phénomène : la dispersion sémantique prouve combien les réalités couvertes sont polymorphes. Une majorité des définitions, certes, décrivent une création d'entreprise due à la perte d'un emploi salarié (Block et Wagner, 2010). Certains articles ont uniquement trait à l'entrepreneuriat de survie (Serviere, 2010), cependant que d'autres évoquent plus généralement l'entrepreneuriat de nécessité comme solution pour échapper aux discriminations subies, par les femmes ou les immigrants par exemple (Clark et Drinkwater, 2000 ; Hammarstedt, 2001 ; Claret et Ruane, 2010).

Dans ces conditions, il nous a semblé important d'analyser plus en détail ce que le concept recouvre dans le champ de recherche actuel et de rendre compte de l'hétérogénéité des profils d'entrepreneur par nécessité en proposant une typologie. La thèse que nous défendons ici est que les entrepreneurs dits de nécessité, de survie, forcés, involontaires, insatisfaits, ou contraints ne sont pas une catégorie homogène, opposable aux entrepreneurs qui poursuivent de lucratives opportunités d'affaires. L'entrepreneuriat de nécessité dépend moins des motivations des individus, qui sont évolutives et multiples, que des situations et du contexte entrepreneurial, car l'environnement de la création par nécessité influence grandement la dialogique porteur de projet-entreprise créée. Or, à ce jour, aucune perspective situationnelle n'est disponible, qui permettrait d'éclairer l'entrepreneuriat de nécessité, non seulement à l'aune des motivations individuelles, mais également au regard du contexte de création et des situations de vie. L'objectif de cet article est, par conséquent, d'identifier, sous le terme générique d'entrepreneur par nécessité, des profils significativement différents les uns des autres, en introduisant en particulier une dimension situationnelle. A cette fin, nous avons entrepris d'établir une typologie des entrepreneurs par nécessité, sur la base de critères issus d'une revue de la littérature. Cette typologie est à destination de la communauté des chercheurs afin d'orienter les travaux futurs, mais aussi des décideurs et du monde politique pour proposer des politiques publiques mieux ciblées, ou des accompagnants et des professionnels $d u$ secteur de l'entrepreneuriat afin d'assurer plus efficacement leur rôle, en analysant dans sa globalité l'évolution de la dialogique entrepreneur-projet de création au regard

RIMHE - Revue Interdisciplinaire Management, Homme \& Entreprise

n²2 - Eté 2016 


\section{Auteurs invités : Une approche typologique de l'entrepreneuriat de nécessité Christel TESSIER-DARGENT, Alain FAYOLLE}

du contexte et du macro-environnement au-delà des seules motivations individuelles. Car, si les profils des entrepreneurs par nécessité s'avèrent contrastés, des accompagnements différenciés et adaptés à chaque profil doivent être envisagés.

\section{De l'hétérogénéité de l'entrepreneuriat de nécessité}

Les sources d'hétérogénéité de l'entrepreneuriat de nécessité peuvent être identifiées par application du cadre d'analyse de Gartner (1985) qui suggère de se focaliser sur quatre dimensions qui décrivent et explicitent toute création d'entreprise : l'individu, l'environnement, l'organisation et le processus. S'agissant de la dimension individuelle, la littérature révèle un ensemble de caractéristiques des entrepreneurs par nécessité, qui reviennent régulièrement dans les travaux (Singh et Denoble, 2003 ; Valeau, 2006 ; Yanniv et Brock, 2012). Ils ont une plus grande aversion aux risques, plus de doutes et plus besoin d'un accompagnement, que les entrepreneurs par opportunité. Ils espèrent retrouver un statut de salarié : le degré de réticence à devenir ou rester entrepreneur est très élevé. Le besoin d'autonomie et de réalisation, le contrôle interne, la propension à prendre des risques et l'auto-efficacité sont faibles. La plupart des travaux (Robichaud, Lebrasseur et Nagarajan, 2010 ; Hernandez, Nunn et Warnecke, 2012) convergent pour conclure à certaines caractéristiques sociodémographiques communes aux entrepreneurs par nécessité, en comparaison avec les entrepreneurs par opportunité : un âge plus élevé, un niveau d'études inférieur, une expérience de travail moins grande, une moindre capacité à identifier et exploiter les opportunités entrepreneuriales, un réseau entrepreneurial limité voire inexistant. Les principaux profils décrits sont les femmes, les seniors, les immigrants, les ruraux, les chômeurs, les individus peu diplômés et en particulier les jeunes, dont le capital social et humain est réputé inférieur. Les motivations de ces entrepreneurs peuvent évoluer dans le temps, en particulier devenir positives si le projet de création se développe. Par ailleurs, les motivations positives et négatives à créer sont en réalité très intriquées, d'ordre culturel, situationnel et psychologique. Ainsi, Beaucage, Laplante et Légarée (2004) montrent dans leur modèle que le passage au travail autonome découle le plus souvent d'une décision motivée à la fois par des aspirations personnelles et professionnelles spécifiques et par des conditions d'emploi précaires et insatisfaisantes: il y a influence combinée des facteurs «pull» et «push», de motivations personnelles et de contraintes économiques et sociales. Concernant l'environnement de la création d'entreprise par nécessité, les données restent contradictoires et non concluantes. Le niveau de chômage, par exemple, joue un rôle indéterminé sur la création par nécessité. Cependant, les pays avec le plus fort taux d'entrepreneuriat de nécessité sont aussi les moins développés économiquement, et ils présentent de forts taux de chômage (Reynolds et al., 2001 ; Wennekers et al., 2005 ; Acs, 2006). Les changements structurels du marché du travail jouent également un rôle prépondérant sur l'accroissement de la création par nécessité, via la précarisation,

RIMHE - Revue Interdisciplinaire Management, Homme \& Entreprise

n²2 - Eté 2016 


\section{Auteurs invités: Une approche typologique de l'entrepreneuriat de nécessité Christel TESSIER-DARGENT, Alain FAYOLLE}

la sous-traitance et la dérégulation. De même, la culture entrepreneuriale du pays favorise le succès des créations d'entreprise, même par nécessité. Enfin et surtout, les politiques publiques «push », réduisant les allocations chômage, obligeant à prendre des emplois, mêmes précaires ou mal rémunérés, sont des facteurs forts contribuant à l'augmentation du nombre d'entrepreneurs par nécessité (Henrekson, 2005). Un niveau élevé de corruption a également un impact «positif» sur la création d'entreprises par nécessité (Mitchell et Campbell, 2009).

Comment caractériser les organisations qui résultent de l'action des entrepreneurs par nécessité ? Les entrepreneurs fortement contraints développent rarement des activités dans un secteur où ils bénéficient d'une expérience, contrairement aux entrepreneurs volontaires. Cet entrepreneuriat est surtout présent dans les secteurs moins complexes, avec peu de barrières à l'entrée, comme les services à la personne ou le secteur informel, réclamant moins d'investissements et plus immédiatement accessibles (Niitykangas, Littunen et Kinnunen, 1998 ; Giacomin et al., 2007). Les entreprises créées sont de petite taille, subissant une forte concurrence des firmes plus grandes et expérimentées du voisinage. Les opportunités poursuivies sont peu rémunératrices (Block et al., 2014 ; Caliendo et al., 2015). Les organisations créées sont aussi le signe d'une hybridité nouvelle des structures, fruit de la précarisation du marché du travail, avec la porosité des frontières entre les différents états représentés par l'emploi, le chômage et l'inactivité (Grüner, 2010 ; Levratto et Serverin, 2012). Il y a dépendance économique et relative indépendance de statut, sous la forme du portage salarial et de l'auto-entrepreneuriat français, de la Ich-AG allemande, du payrolling aux Pays Bas et en Belgique, des umbrella companies au Royaume Uni ou encore des egenanställning en Suède et des coopératives espagnoles. Les avis divergent quant à l'impact économique de ces organisations créées par nécessité et à leur pérennité. Ainsi en 2004, Maritz affirme sur la base des données GEM qu'il y a un lien positif entre entrepreneuriat de nécessité et croissance (Maritz, 2004). Cependant Wong, Ho et Autio (2005) concluent qu'il n'y a pas de différence en termes d'impact sur la croissance économique entre entrepreneuriat de nécessité et d'opportunité. En 2006, Acs démontre en utilisant des statistiques GEM également, que la création d'entreprise par nécessité n'a pas d'effet sur le développement économique d'un pays, contrairement à l'effet d'entraînement et de croissance positif engendré par l'entrepreneuriat d'opportunité (Acs, 2006).

Pour Sternberg (2011), les entrepreneurs par nécessité, même s'ils sont minoritaires et qu'ils réussissent globalement moins bien que les entrepreneurs par opportunité, ont néanmoins une contribution économique positive, car ils créent des emplois, innovent, exportent et développent la culture entrepreneuriale des pays. D'une manière générale, on pourrait considérer que les processus de création d'entreprise caractéristiques de l'entrepreneuriat de nécessité oscillent entre désenchantement et résilience. Kodithuwakku et Rosa (2002) démontrent que les processus entrepreneuriaux sont 


\section{Auteurs invités : Une approche typologique de l'entrepreneuriat de nécessité Christel TESSIER-DARGENT, Alain FAYOLLE}

déterminants dans la réussite des entrepreneurs développant leur entreprise avec de faibles ressources et dans des environnements peu prometteurs et extrêmement contraints. Les entrepreneurs par nécessité rencontrant le succès dans ces conditions difficiles ne sont pas plus innovants pour identifier les opportunités d'affaires. Par contre, ils sont plus créatifs et persévérants pour mobiliser des ressources rares. Ils exploitent plus efficacement leur réseau social et leurs contacts pour accumuler du capital, et possèdent de bonnes capacités de gestion. Hernandez (2006, p.337) affirme cependant que la dimension «décision » est le plus souvent absente du processus entrepreneurial, en particulier par nécessité : «L'individu devient entrepreneur mais sans véritablement l'avoir décidé. Il apparaît plus souvent comme un agent pris dans un contexte qui le pousse vers l'entrepreneuriat que réellement comme un acteur décidant en toute connaissance de cause de créer sa propre organisation ». Mandják, Bárdos, Neuman-Bódi, Németh et Simon (2011) décrivent les entrepreneurs par nécessité comme des héros tragiques. Mus par des « déplacements » (échec scolaire, divorce, licenciement), ils sont forcés de faire quelque chose pour lequel ils ne sont ni préparés ni motivés. Et malgré tout, ils mettent toute leur énergie à accomplir cette tâche, sachant qu'ils sont, en raison des circonstances, quasiment voués à l'échec. Anderson (2005) a démontré que les chômeurs et les inactifs qui créent leur entreprise changent généralement de statut pour retrouver le chômage et l'inactivité, plutôt que pour partir vers des emplois salariés, ce qui est un marqueur d'échec de ce type de processus entrepreneurial. Block et Koellinger (2009) démontrent d'ailleurs que les entrepreneurs par nécessité sont significativement moins satisfaits par leur choix occupationnel que les entrepreneurs par opportunité. Gérer sa société au quotidien, trouver les ressources pour la pérenniser est souvent source de désenchantement.

Néanmoins, il ne faut pas sous-estimer, dans ces processus, le rôle positif de la résilience : se lancer dans un projet est une façon de redonner à sa vie, à partir de soi, une vraie cohérence ; cela alimente estime de soi et confiance en soi, surtout pour les victimes de l'exclusion, comme les seniors ou les immigrants. Quelques travaux francophones (Bernard, 2008 ; Brasseur, 2010) soulignent l'intérêt de l'entrepreneuriat de nécessité comme mode d'insertion professionnelle alternatif au recrutement classique, voire comme cursus permettant aux plus démunis de sortir de l'exclusion et de rebondir.

\section{Méthodologie d'élaboration de la typologie}

Une typologie est un «ensemble de configurations conceptuelles définies a priori à partir d'attributs multiples et [qui] suit la logique wébérienne d'idéaltype en s'appuyant sur une approche déductive et qualitative » (Borgès Da Silva, 2013, p.635). Pour déterminer qui sont réellement les entrepreneurs par nécessité, nous avons retenu cette approche théorique et entrepris de construire une typologie, par l'identification des caractéristiques spécifiques à l'entrepreneuriat de nécessité prédéterminées

RIMHE - Revue Interdisciplinaire Management, Homme \& Entreprise

n²2 - Eté 2016 


\section{Auteurs invités : Une approche typologique de l'entrepreneuriat de nécessité Christel TESSIER-DARGENT, Alain FAYOLLE}

théoriquement sur la base d'une revue de la littérature internationale, qui a fourni l'ensemble des attributs, que nous nommerons descripteurs. Pour construire notre typologie, nous avons analysé la littérature spécifique à l'entrepreneuriat de nécessité, afin d'identifier l'ensemble des caractéristiques convoquées dans ce champ de recherche. Cette revue tend vers l'exhaustivité et est transdisciplinaire et multilingue (français, anglais, allemand et espagnol). L'analyse de littérature que nous avons effectuée et dont nous présentons les étapes ci-dessous est une analyse de contenu qualitative qui, sans les appliquer formellement, s'inspire très largement des principes et techniques de l'analyse systématique de littérature (Systematic Literature Review, SLR).

Dans un premier temps, nous avons déterminé une liste de mots-clefs permettant de couvrir le champ de l'entrepreneuriat de nécessité Plusieurs étapes se sont ensuite succédées pour constituer le corpus étudié : a) nous avons interrogé Google Scholar et les bases de données pertinentes (Business Source Complete, Proquest, Ebsco, Winley library, Cairn info, Emerald, Science Direct, New Economics Papers on Entrepreneurship) sur les mots-clefs en français et en anglais d'entrepreneur ou entrepreneuriat par nécessité, auto-entrepreneuriat, entrepreneuriat subi ou contraint ou forcé, entrepreneurs malgré-eux, motivations push, création par nécessité, effet refuge, necessity entrepreneur(ship), unemployment and entrepreneurship, self-employment and necessity or constraint or precarity, reluctant or involuntary or forced or constrained or pushed entrepreneur(ship), refugee, push motivations ; b) nous avons mis en place une stratégie de veille, sur Google Scholar et les autres bases de données citées, permettant d'obtenir quotidiennement les textes incluant un des mots-clefs fournis. La pertinence et l'intérêt des documents ont été déterminés à la lecture du texte intégral; c) nous avons effectué des recherches systématiques dans les principaux journaux classés en entrepreneuriat (classement HCERES) ; d) nous avons revu les papiers des principales conférences en entrepreneuriat; e) nous avons contacté la communauté des chercheurs travaillant dans le domaine de l'entrepreneuriat de nécessité, afin d'obtenir d'éventuelles études et résultats non encore publiés ; f) enfin nous avons fait une recherche bibliographique approfondie pour les auteurs particulièrement prolifiques et pertinents dans ce champ de recherche, tels Rosa, Block ou Wagner.

Nous avons adopté une démarche transdisciplinaire : a) nous avons lu les articles issus de revues de management et gestion, psychologie, sociologie, anthropologie économique et culturelle, droit, économie politique, stratégie, marketing, finance, géographie et histoire; b) les bibliographies des articles sélectionnés ont été exploitées, et tous les référencements croisés ont été explorés; d) nous avons également consulté les manuels et autres ouvrages de référence.

Trois critères ont permis de sélectionner les publications sur lesquelles nous nous sommes appuyés. Nous avons dans un premier temps retenu, sur le thème de

RIMHE - Revue Interdisciplinaire Management, Homme \& Entreprise

n²2 - Eté 2016 


\section{Auteurs invités : Une approche typologique de l'entrepreneuriat de nécessité Christel TESSIER-DARGENT, Alain FAYOLLE}

l'entrepreneuriat de nécessité, les publications expertisées par des comités éditoriaux. Les actes de colloques ou conférences, les ouvrages collectifs ou individuels, ainsi que les papiers de recherche et les thèses de doctorat ont été conservés pour complément dans la mesure de leur originalité et de leur fiabilité, compte tenu de la contemporanéité du sujet. Les articles de revues classées et les ouvrages référencés dans des sciences connexes, dont les thématiques se centraient sur le champ de l'entrepreneuriat de nécessité, ont également été retenus. Un corpus de 240 références académiques a ainsi été obtenu. Seuls les articles dont le cœur de la réflexion portait sur l'entrepreneuriat de nécessité ont été conservés. En particulier, les articles sur d'autres thèmes (entrepreneuriat par les immigrés, entrepreneuriat social, etc.), souvent élaborés à partir des données GEM et n'évoquant que de façon anecdotique le cas des entrepreneurs par nécessité, ont été écartés. Après lecture intégrale et rédaction d'un résumé, une base de données a été constituée, détaillant chaque article (discipline, cadre théorique, méthode, apports, limites, définition de l'entrepreneuriat de nécessité). Les caractéristiques associées à l'entrepreneuriat de nécessité ont ainsi été relevées dans la littérature spécialisée, puis classées en catégorie. La différenciation proposée par Mandják, Bárdos, Neuman-Bódi, Németh et Simon (2011), entre création par nécessité engendrée par des facteurs externes, liés à l'environnement, tels le chômage ou la dérèglementation du marché du travail, et création par nécessité due à des facteurs internes à l'individu, comme l'insatisfaction au travail, a notamment permis un premier tri des caractéristiques recensées. Sur la base des caractéristiques identifiées dans la littérature, nous avons construit une typologie en huit profils descriptifs. Nous avons procédé par segmentation et congruence sur cette liste de facteurs, afin d'obtenir des profils d'entrepreneurs par nécessité identifiables et différenciés. Chaque profil décrit un groupe homogène d'individus aux caractéristiques proches. Ces types sont pertinents, c'est-à-dire utilisables, mesurables et accessibles, afin de les rendre opérationnels. Cette pratique de la segmentation met en relief de manière contrastée le degré d'hétérogénéité pouvant exister, au-delà d'une homogénéité de prime abord entre sous-ensembles.

\section{Descripteurs de l'entrepreneuriat de nécessité et typologie associée}

Nous avons pu identifier des descripteurs de l'entrepreneuriat de nécessité, c'est-à-dire des caractéristiques qu'il est possible d'associer aux entrepreneurs par nécessité. A partir de ces descripteurs, nous avons construit notre typologie reposant sur huit profils différenciés d'entrepreneurs par nécessité.

\subsection{Des descripteurs regroupés en trois axes}

Les caractéristiques décrivant les entrepreneurs par nécessité peuvent être regroupées selon trois axes : 1) descripteurs externes, 2) descripteurs intrinsèques discriminants et 3) descripteurs internes, d'ordre psychologique. Nous avons ensuite opéré un sous

RIMHE - Revue Interdisciplinaire Management, Homme \& Entreprise

n²2 - Eté 2016 


\section{Auteurs invités : Une approche typologique de l'entrepreneuriat de nécessité Christel TESSIER-DARGENT, Alain FAYOLLE}

classement des caractéristiques externes, selon qu'elles relèvent de situations contraignantes d'ordre économique, social, ou légal. Le premier axe décrit les caractéristiques externes, difficilement modifiables, telles que les différentes situations de vie contraignantes évoquées par Fayolle et Nakara (2012). Ces attributs sont objectifs, mesurables liés à l'environnement ou à une trajectoire de vie. Concernant les situations économiques contraignantes tout d'abord, il s'agit du chômage, de l'intérim à répétition et de la précarité salariale, de la dépendance aux minima sociaux, d'une préretraite ou d'une retraite proche ou en deçà du seuil de pauvreté. Pour les situations légales contraignantes, les facteurs relevés dans la littérature mentionnent le travail au noir, qui implique de prendre un statut juridique pour sortir de l'économie informelle et développer une activité, ainsi que la sous-traitance souvent imposée par l'employeur. Enfin, un nouveau lieu de vie sans opportunité professionnelle est une forme de situation sociale contraignante, tout comme peut l'être l'obligation de reprendre l'entreprise familiale ou la présence de mauvaises conditions de travail.

Le deuxième axe recense les profils discriminants des individus. Ces caractéristiques intrinsèques, non modifiables, sont les stigmates décrits par Alter (2012) et étudiés dans de nombreux travaux dont ceux de Clark et Drinkwater (2000) ou Hammarstedt (2001). Une intervention des pouvoirs publics au niveau sociétal serait nécessaire pour changer le regard porté sur différentes catégories d'individus, dont le capital humain est réputé faible. Ces caractéristiques décrivent différents groupes sociaux stigmatisés : minorités ethniques, immigrés ne maîtrisant pas la langue du pays hôte, parents isolés, seniors, jeunes, en particulier sans diplôme ni qualification, et enfin personnes handicapées ou connaissant des problèmes de santé. Leurs stigmates ajoutent aussi des contraintes à ces individus salariés comme entrepreneurs et créent des freins supplémentaires, des difficultés à obtenir des prêts bancaires ou à constituer des réseaux d'affaires.

Le troisième axe regroupe les caractéristiques internes, psychologiques et modifiables, de l'ordre des traits, croyances, compétences et surtout motivations, tels qu'étudiées dans les rapports GEM ou les travaux de Singh et Denoble (2003) ou Tyszka et Cieslik (2011). Il serait possible d'intervenir sur ces facteurs car ils sont subjectifs et évolutifs. Difficilement mesurables, ils sont pourtant à l'origine de la plupart des études quantitatives, dont celles d'Amit et Mueller (1995) et Robichaud, Lebrasseur et Nagarajan (2010). Ces travaux s'appuient sur une perception à un instant donné, recueillie auprès des entrepreneurs par des enquêteurs extérieurs, sous la forme d'une unique question fermée portant sur la motivation à créer. Ces descripteurs internes incluent également les traits psychologiques, tels qu'un faible besoin d'autonomie ou une forte aversion aux risques, un «locus of control » externe, une faible estime de soi, peu de confiance en ses capacités propres. Les croyances incluent une faible autoefficacité. Les motivations sont dites extrinsèques élevées, intrinsèques faibles, et contextuelles négatives. Les compétences entrepreneuriales décrivent des capacités

RIMHE - Revue Interdisciplinaire Management, Homme \& Entreprise

n²2 - Eté 2016 


\section{Auteurs invités : Une approche typologique de l'entrepreneuriat de nécessité Christel TESSIER-DARGENT, Alain FAYOLLE}

managériales faibles, une insuffisante capacité à identifier et exploiter les opportunités entrepreneuriales, à mobiliser des ressources et à créer une organisation. Ces facteurs sont supposés être communs à la plupart des entrepreneurs par nécessité, de façon plus ou moins exacerbée sans que des études corroborent ces a priori.

Tableau 1 : Les trois axes de caractéristiques décrivant les entrepreneurs par nécessité

\begin{tabular}{|c|c|c|}
\hline $\begin{array}{c}\text { Caractéristiques externes liées à des } \\
\text { situations contraignantes }\end{array}$ & $\begin{array}{l}\text { Caractéristiques } \\
\text { intrinsèques discriminantes }\end{array}$ & $\begin{array}{l}\text { Caractéristiques internes } \\
\text { d'ordre psychologique }\end{array}$ \\
\hline $\begin{array}{l}\text { Economique } \\
\text { - chômage (fin de droits, longue } \\
\text { durée) } \\
\text { - intérim à répétition, précarité } \\
\text { salariale } \\
\text { - minima sociaux } \\
\text { - préretraite ou retraite proche/en } \\
\text { deçà du seuil de pauvreté } \\
\text { Légal } \\
\text { - travail au noir } \\
\text { - sous-traitance imposée } \\
\text { Social } \\
\text { - nouveau lieu de vie } \\
\text { - obligation de reprendre } \\
\text { l'entreprise familiale ou de } \\
\text { l'associé } \\
\text { - mauvaises conditions de travail }\end{array}$ & $\begin{array}{l}\text { - femme } \\
\text { - minorité ethnique } \\
\text { immigré, non maîtrise de la } \\
\text { langue du pays hôte } \\
\text { - parent isolé } \\
\text { - senior } \\
\text { - jeune, sans diplôme ou } \\
\text { qualification } \\
\text { - handicapé, problème de } \\
\text { santé } \\
\text { - interdit bancaire }\end{array}$ & $\begin{array}{l}\text { - absence de motivation } \\
\text { positive à créer } \\
\text { - « locus of control » } \\
\text { externe } \\
\text { - aversion forte au risque } \\
\text { - absence de culture } \\
\text { entrepreneuriale et de } \\
\text { référent (réseau, tuteur, } \\
\text { famille, accompagnement) } \\
\text { - faible estime de soi } \\
\text { - peu de confiance en ses } \\
\text { capacités propres }\end{array}$ \\
\hline
\end{tabular}

\subsection{Une typologie des entrepreneurs par nécessité}

Sur la base de l'ensemble des descripteurs recensés dans la littérature, nous avons identifié huit profils d'entrepreneurs par nécessité (voir tableau 2). Les verbatim mentionnés en illustration sont issus d'une enquête réalisée auprès de 1000 entrepreneurs, interrogés entre septembre 1998 et octobre 2013 par la couveuse d'entreprise COSENS ${ }^{19}$ basée à Marseille. Six des profils correspondent à des caractéristiques externes non modifiables liées au microenvironnement et à des situations de vie particulières et contraignantes. Un profil représente les facteurs intrinsèques non modifiables liés aux discriminations supportées par certains groupes d'individus. Le dernier profil représente les descripteurs internes d'ordre psychologique, en particulier la motivation. Pour passer des descripteurs identifiés dans la littérature aux profils définis dans la typologie, nous avons opéré par congruence au sens de Mintzberg (1980), afin d'éviter les redondances engendrées en conservant tous les profils théoriques identifiés.

\footnotetext{
${ }^{19}$ http://www.cosens.fr
}

RIMHE - Revue Interdisciplinaire Management, Homme \& Entreprise

n²2 - Eté 2016 


\section{Auteurs invités : Une approche typologique de l'entrepreneuriat de nécessité Christel TESSIER-DARGENT, Alain FAYOLLE}

Tableau 2: Typologie en huit profils des entrepreneurs par nécessité.

\begin{tabular}{|c|c|c|c|}
\hline & & Définition & Illustration \\
\hline \multirow[t]{2}{*}{ 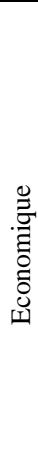 } & 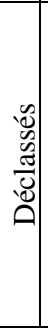 & $\begin{array}{l}\text { Maintenus, contre leur } \\
\text { souhait, hors du marché du } \\
\text { travail salarié, malgré } \\
\text { diplôme et expérience. Ils } \\
\text { pourraient trouver un } \\
\text { emploi ne correspondant } \\
\text { pas à leurs attentes et } \\
\text { qualifications, et préfèrent } \\
\text { créer leur entreprise. }\end{array}$ & $\begin{array}{l}\text { Monsieur B., plus de } 50 \text { ans, ancien ingénieur, n'avait pas } \\
\text { prévu de se tourner vers la création d'entreprise. Mais } \\
\text { après plusieurs années de chômage, il doit se rendre à } \\
\text { l'évidence : le monde du travail est fermé pour lui. La } \\
\text { seule manière de travailler jusqu'à sa retraite est de créer } \\
\text { son propre emploi. Il envisage le nettoyage de tombes, } \\
\text { l'aide à domicile, puis se tourne vers l'entretien d'espaces } \\
\text { verts pour les entreprises (rapport Discrimination et } \\
\text { création d'entreprise, ALEXIS Alsace, 2008, p.44) }\end{array}$ \\
\hline & |ص: & $\begin{array}{l}\text { Véritables entrepreneurs de } \\
\text { survie, ils créent pour } \\
\text { assurer la subsistance de } \\
\text { leur foyer. }\end{array}$ & $\begin{array}{l}\text { Verbatim : «Là, où j'en suis, je ne vois rien d'autre à faire } \\
\text { pour assurer mon existence matérielle»; «Je crée mon } \\
\text { entreprise de coiffure à domicile pour sortir de la } \\
\text { précarité ». }\end{array}$ \\
\hline \multirow[t]{2}{*}{ 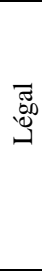 } & 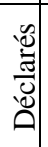 & $\begin{array}{l}\text { Contraints de légaliser une } \\
\text { activité } \\
\text { fructueuse pour en assurer } \\
\text { le développement. }\end{array}$ & $\begin{array}{l}\text { Verbatim : «J'ai de plus en plus de demande dans la } \\
\text { légalité.»; «Pour pouvoir facturer mes prestations. »; } \\
\text { «Assurer la pérennité de mon activité, me structurer } \\
\text { juridiquement. » }\end{array}$ \\
\hline & 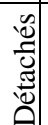 & $\begin{array}{l}\text { Contraints par leur } \\
\text { employeur de prendre un } \\
\text { statut de sous-traitant. }\end{array}$ & $\begin{array}{l}\text { S., ingénieure, a « rencontré une urbaniste qui se lance». } \\
\text { Pour être embauchée, elle a dû accepter de devenir auto- } \\
\text { entrepreneur. (Vivant, 2013, p.5) }\end{array}$ \\
\hline \multirow{2}{*}{ 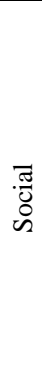 } & 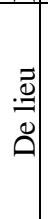 & $\begin{array}{l}\text { Contraints par un lieu } \\
\text { d'habitation, ils créent car } \\
\text { ils ne retrouvent pas } \\
\text { d'emploi salarié } \\
\text { correspondant à leurs } \\
\text { attentes et qualifications. }\end{array}$ & $\begin{array}{l}\text { Une quarantenaire, mère de deux enfants, a travaillé de } \\
\text { nombreuses années comme assistante maternelle. Après } \\
\text { un déménagement, elle a dû accepter des emplois } \\
\text { précaires, puis est finalement restée deux ans au chômage } \\
\text { avant de créer son emploi. (Kantola, 2013, p.188) }\end{array}$ \\
\hline & 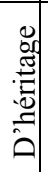 & $\begin{array}{l}\text { Contraints de reprendre } \\
\text { l'entreprise familiale ou de } \\
\text { leur collaborateur. }\end{array}$ & $\begin{array}{l}\text { Verbatim: «Je n'ai pas d'autre choix que de racheter le } \\
\text { garage familial et de poursuivre l'affaire. J'aurais voulu } \\
\text { être coiffeur.» }\end{array}$ \\
\hline 䓵 & |ֶ:ڤ & $\begin{array}{l}\text { Maintenus, contre leur } \\
\text { souhait, hors du marché du } \\
\text { travail salarié, en raison de } \\
\text { stigmates, ils créent pour } \\
\text { assurer leur développement } \\
\text { professionnel. }\end{array}$ & $\begin{array}{l}\text { Madame L., immigrée : «Une femme arabe dans un poste } \\
\text { à responsabilité, ça ne passe pas... Je n'ai pas réussi à } \\
\text { l'avoir par le travail, c'est pour ça que j'ai créé mon } \\
\text { entreprise. Jusqu'à il y a deux ans, je n'avais jamais voulu } \\
\text { créer une entreprise. » (rapport Discrimination et création } \\
\text { d'entreprise, ALEXIS Lorraine, 2008, p.47). }\end{array}$ \\
\hline 蛋 & 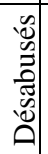 & $\begin{array}{l}\text { Désireux de fuir une } \\
\text { expérience très négative et } \\
\text { frustrante du salariat. }\end{array}$ & $\begin{array}{l}\text { Verbatim: «Je suis écœuré de travailler pour des patrons } \\
\text { incompétents, sans perspective d'évolution de carrière, } \\
\text { avec des heures supplémentaires non payées». }\end{array}$ \\
\hline
\end{tabular}

RIMHE - Revue Interdisciplinaire Management, Homme \& Entreprise

n²2 - Eté 2016 


\section{Auteurs invités : Une approche typologique de l'entrepreneuriat de nécessité Christel TESSIER-DARGENT, Alain FAYOLLE}

Chaque profil est une synthèse des descripteurs signifiants. Par exemple, les différents types de discrimination décrits correspondent à un profil unique, en ce que l'enjeu n'est pas la forme prise par le stigmate mais bien sa résultante, homogène en termes de conséquences sur le choix du processus entrepreneurial et son déroulement. La simplification permet de se focaliser sur l'élément principal, à savoir l'impact d'un type de profil d'entrepreneur par nécessité en termes de contraintes à la création. De même, pour les descripteurs internes, compte-tenu de la faiblesse des études en la matière et de l'enjeu primordial de l'accompagnement psychologique, un profil unique a été retenu, basé sur la motivation négative et extrinsèque à créer, seul facteur psychologique réellement étudié à ce jour chez les entrepreneurs par nécessité. Pour les critères économiques enfin, deux niveaux significatifs, celui du déclassement économique et celui de la survie, permettent d'agréger sous deux profils remarquables et pertinents, le continuum d'individus qui créent, poussés par des raisons économiques.

Les «déclassés », qui ne trouvent pas leur place dans la société en tant que salariés, sont des seniors préretraités, des cadres licenciés après restructuration, des jeunes précaires, tous professionnels diplômés et expérimentés maintenus hors du marché $\mathrm{du}$ travail contre leur volonté (Yaniv et Brock, 2012 ; Bell et Rutherford, 2013).

Les «démunis » sont de véritables entrepreneurs de survie (Clare et Ruane, 2010 ; Carlsrud et Brännback, 2011). La création d'entreprise vise ici la subsistance de l'individu et de ses proches. Il concerne, de fait, plutôt les pays dits en développement ou à économie de facteurs. Les individus créent pour subvenir à leurs besoins primaires, dans des économies souvent marquées par la corruption, l'économie souterraine, une forte hiérarchisation de la société, des infrastructures peu développées, l'absence de filets sécuritaires sociaux. Néanmoins, quand le chômage augmente, que les plus pauvres se paupérisent encore, en temps de crise par exemple, ce phénomène atteint aussi les pays dits développés.

Les «déclarés » créent une entreprise pour légaliser une activité fructueuse mais pratiquée de façon informelle. Ces cas révèlent, en creux, le peu d'attirance des entrepreneurs pour un statut légal s'il est possible de s'en passer, compte-tenu de fortes charges sociales, d'une taxation importante et de lourdes contraintes administratives. Selon le rapport «Le régime de l'auto-entrepreneur, bilan après une année de mise en œuvre » rédigé par la Direction Générale de la Compétitivité de l'Industrie et des Services (2009, p.74) : «23\% des auto-entrepreneurs affirment avoir saisi l'occasion de professionnaliser une activité déjà exercée ». Cette catégorie d'entrepreneurs par nécessité concourt à réduire le travail illégal et non déclaré, avec sa cohorte de dangers et d'inconvénients, pour les individus, les clients et la société en général. Ces entrepreneurs, dans la mesure où la transition de modèle économique est viabilisée par des allègements de charges sociales par exemple, sont plutôt bénéfiques au développement économique.

RIMHE - Revue Interdisciplinaire Management, Homme \& Entreprise

n²2 - Eté 2016 


\section{Auteurs invités: Une approche typologique de l'entrepreneuriat de nécessité Christel TESSIER-DARGENT, Alain FAYOLLE}

Les «détachés » sont les individus poussés vers l'entrepreneuriat par leurs employeurs; sous-traitants dans la presse, le BTP ou l'expertise comptable par exemple (Beaucage et Bellemare, 2007). Il s'agit d'un type d'entrepreneur par nécessité très spécifique lié à l'assouplissement du marché du travail. Ces individus adoptent un statut d'indépendant et créent leur propre structure pour trouver ou conserver un travail. Les entreprises allègent ainsi leurs charges sociales et retrouvent de la flexibilité, en externalisant risques et incertitudes. Ces entrepreneurs n'ont aucune motivation pour ce faire et dépendent le plus souvent d'un seul employeur. Il s'agit d'une "zone grise » d'emploi, utilisant les interstices du droit du travail. Ce problème doit être abordé sous cet angle pour ne pas favoriser une précarité nuisible aux employés (Girard, 2007).

Les «de lieu»sont les personnes contraintes par un impératif géographique et ne trouvant pas d'emploi (Kantola, 2013). Ces créations d'entreprise sont souvent des étapes transitoires, assouplies par les possibilités technologiques et organisationnelles de télétravail. Ces parcours demeurent difficiles pour celles et ceux qui les empruntent. Les «d'héritage » sont contraints à reprendre l'entreprise familiale ou celle de leur associé (Giacomin et al., 2007 ; Bhola et al., 2006). Ces entrepreneurs se voient imposer l'entrepreneuriat, sans y être réellement préparés ou le souhaiter. Ce type d'entrepreneurs par nécessité bénéficie généralement de conditions favorables. Néanmoins les motivations et les compétences peuvent faire défaut. Ce parcours peut mener à l'entrepreneuriat d'opportunité et au développement d'innovation ou, au contraire, représenter un fardeau de longues années durant.

Les « discriminés » sont ceux qui ne parviennent pas à trouver d'emploi en raison de leur caractéristique, ce qu'Alter (2012, p.26) nomme «stigmate»: minorités ethniques, jeunes sans diplôme, mères célibataires, femmes de plus de quarante ans divorcées avec enfants (Girard, 2007; Nakara et Fayolle, 2013). Ils ont en commun de subir des discriminations à l'emploi et d'avoir un capital humain et social réputé faible. Ils sont souvent fragilisés et cumulent les facteurs contraignants. Néanmoins, correctement accompagnés, ils peuvent utiliser ce parcours de création comme une opportunité pour rebondir et se réinsérer dans le monde du travail. Dans leur cas, l'adaptation des politiques publiques et l'accompagnement sont particulièrement importants, la phase de création ne devant le plus souvent rester que transitoire pour être fructueuse. Il s'agit de donner une expérience ré-exploitable et de la confiance en soi à ces personnes. De nombreux articles (Orhan, 2001; Fraser, 2009 ; Maâlaoui et Germon, 2014) témoignent cependant que ces entrepreneurs subissent également des discriminations dans leur parcours de porteurs de projet.

Les «désabusés » sont les personnes qui fuient le salariat et des conditions de travail pénibles, stress ou harcèlement (Kantola, 2013), emploi du temps contraignant, absence de perspectives de carrière. Dans cette catégorie, les compétences entrepreneuriales, expériences professionnelles, réseaux d'appui, varient énormément.

RIMHE - Revue Interdisciplinaire Management, Homme \& Entreprise

n²2 - Eté 2016 


\section{Auteurs invités : Une approche typologique de l'entrepreneuriat de nécessité Christel TESSIER-DARGENT, Alain FAYOLLE}

\section{Conclusion}

A partir d'une revue de la littérature sur le thème de l'entrepreneuriat de nécessité, nous avons construit une typologie situationnelle incluant une perspective dynamique. Cette typologie est originale en ce qu'elle permet de nuancer une représentation très souvent dichotomique et réductrice de l'entrepreneuriat opposant nécessité et opportunité, en l'étoffant par des profils affinés d'entrepreneurs par nécessité, ainsi que le suggéraient Giacomin, Guyot, Janssen et Lohest (2007). Cependant, comme toute typologie, celle proposée ici pour les entrepreneurs par nécessité est critiquable et imparfaite. Ainsi, les seniors, classés parmi les « déclassés », auraient pu également rejoindre la catégorie des "discriminés », comme en témoignent leurs discours et certains travaux sur le sujet (Smeaton, 2003 ; Bell et Rutherford, 2013). Le choix a été fait de les maintenir dans la catégorie des «déclassés », car le facteur de contrainte principal est lié à l'impossibilité de trouver un emploi malgré l'expérience et les diplômes, un individu devant figurer dans une catégorie unique à un moment donné.

De même, tous les types identifiés ne sont pas de même nature. La nécessité suggérée par le cas des entrepreneurs d'héritage est sans commune mesure avec les entrepreneurs de survie. Pourtant, tous deux sont agrégés dans les études quantitatives, et il était nécessaire de mettre ce point en exergue et d'intégrer à la typologie tous les types identifiés dans la littérature. Ces individus ont en commun d'éprouver une faible satisfaction à créer, de développer peu leur entreprise, de souhaiter redevenir salariés et d'avoir un faible niveau d'autoréalisation.

Un regard réflexif permet de mettre en perspective notre proposition de typologie. Son objectif principal énoncé était d'éclairer l'oxymore apparent que peut représenter l'entrepreneuriat de nécessité. "La revue de littérature doit contribuer à montrer que les grilles de lecture partagées ne sont plus opératives, qu'au lieu de faciliter la compréhension et la lecture du monde, elles nous rendent aveugles et nous empêchent de voir l'essentiel » (Bureau, 2011, p.69). En cela, notre typologie souligne la grande diversité des profils d'entrepreneurs par nécessité, l'importance du contexte et des situations de vie contingentes au-delà de la seule explication motivationnelle qui masque une réalité beaucoup plus complexe à appréhender. Ces huit types d'entrepreneurs, malgré des proximités apparentes, sont assez différents des profils évoqués dans les recherches antérieures sur l'entrepreneuriat en général (Singh et Denoble, 2003 ; Girard, 2007 ; Schoar, 2009 ; Legrand, Stervinou et Lemaître 2012). Il est donc possible de considérer qu'il y a une relative spécificité de l'entrepreneuriat par nécessité, à la fois dans sa globalité et dans les sous-catégories qu'il est possible d'établir.

Cette typologie des entrepreneurs par nécessité invite à des approches spécifiques en matière d'accompagnement, de formation, de dispositifs de financement et de politiques publiques. Ainsi, il serait probablement utile de distinguer très clairement,

RIMHE - Revue Interdisciplinaire Management, Homme \& Entreprise

n²2 - Eté 2016

- 87 - 


\section{Auteurs invités: Une approche typologique de l'entrepreneuriat de nécessité Christel TESSIER-DARGENT, Alain FAYOLLE}

dans les politiques publiques, les objectifs d'aide sociale et économique, reposant sur des facteurs de contrainte objectifs tels les revenus, la structure familiale, les diplômes, les facteurs de stigmatisation par exemple, et les objectifs d'accompagnement psychologique, liés à des caractéristiques subjectives, telles la motivation, la confiance en soi, l'aversion aux risques. En l'absence de désirabilité et/ou d'alternative, les individus sont fragilisés, mais un certain nombre d'outils peuvent être mobilisés pour rendre cette expérience, même si elle s'avère non pérenne, du moins plus fructueuse. Du point de vue de l'accompagnement par exemple, il conviendrait d'éviter la généralisation par souci d'économie, en croyant s'adresser à une catégorie supposée homogène. Il serait aussi bénéfique de changer la dénomination stigmatisante d' «entrepreneur par nécessité », et de repérer plutôt, pour chaque individu, les caractéristiques propres qui le contraignent.

Pour pousser plus loin la connaissance, une taxonomie permettrait de réaliser une confirmation statistique, si possible sur un échantillon international tel que celui fourni par l'enquête GEM. Une des voies de recherche futures parmi les plus prometteuses consiste à tenter de mettre en évidence les liens entre les types d'entrepreneurs par nécessité obtenus ici et la performance de leurs firmes, notamment en termes de survie et de croissance. A priori, les « déclassés », « de lieu », « déclarés » et «d'héritage », sont mieux armés et devraient mieux réussir que les «démunis », «détachés », « discriminés » et « désabusés ». Une piste de recherche pourrait se résumer d'ailleurs à dresser une grille d'analyse pragmatique des facteurs de nécessité, et de leurs répercussions sur la dialogique «entrepreneur par nécessité/projet de création ».

Une réflexion de nature épistémologique et méthodologique serait également utile. Est-ce que le concept d'entrepreneuriat par nécessité est un bon outil pour nous aider à comprendre ce que la recherche empirique nous donne à voir ? Correspond-il bien à ce que celui qui l'utilise cherche à dire ? Garde-t-il le même sens d'un chercheur à l'autre, voire chez le même auteur?

Par ailleurs, pour dépasser une potentielle impasse d'ordre conceptuel, il semblerait utile de s'intéresser moins à l'individu et plus à la dialogique projet-créateur. Puisqu'un glissement semble pouvoir s'opérer, des motivations individuelles négatives à créer, à un faisceau de contraintes impliquant la création par nécessité, il paraît nécessaire de se pencher sur l'environnement, les situations de vie et le contexte entrepreneurial, pour en mesurer l'impact sur les processus entrepreneuriaux par nécessité. Deux axes se dégagent alors, l'un se focalisant sur l'environnement contraignant de l'entrepreneuriat de nécessité, l'autre appelant une étude de la spécificité des processus entrepreneuriaux par nécessité. 


\section{Auteurs invités : Une approche typologique de l'entrepreneuriat de nécessité}

Christel TESSIER-DARGENT, Alain FAYOLLE

\section{Références bibliographiques}

Abdlenour S., Lambert A. (2014), L'entreprise de soi, un nouveau mode de gestion politique des classes populaires ?, Genèses, vol. 2, n 95 , p. 27-48.

Acs Z.J. (2006), How is entrepreneurship good for economic growth?, Innovations, vol. $1, \mathrm{n}^{\circ} 1$, p. 97-107.

Acs Z.J., Varga A. (2005), Entrepreneurship, Agglomeration and Technological Change, Small Business Economics, vol. 24, n 3, p. 323-334.

Alter N. (2012), La force de la différence; itinéraire de patrons atypiques, Paris, Presses Universitaires de France.

Amit R., Mueller E. (1995), Push and pull Entrepreneurship, Journal of Small Business and Entrepreneurship, vol. 12, $\mathrm{n}^{\circ}$ 4, p. 64-80.

Anderson A. R. (2005), Enacted Metaphor: The Theatricality of the Entrepreneurial Process, International Small Business Journal, vol. 23, p. 587-603.

Beaucage A., Laplante N., Legare R. (2004), Le passage au travail autonome : Choix imposé ou choix qui s'impose ?, Relations industrielles, vol. 59, $\mathrm{n}^{\circ}$ 2, p. 345-378.

Beaucage A., Bellemare G. (2007), La diversité du succès des travailleurs autonomes, Recherches sociographiques, vol. 48, $\mathrm{n}^{\circ} 2, \mathrm{p} .11-36$.

Bell D., Rutherford A. (2013), Older Workers and Working Time, discussion paper $\mathrm{n}^{\circ} 7546$, Institute for the Study of Labor (IZA), Bonn.

Bergmann H., Sternberg R. (2007), The changing face of entrepreneurship in Germany, Small Business Economics, vol. 28, p. 205-221.

Bernard M.J. (2008), L'entrepreneuriat comme un processus de résilience. Les bases d'un dialogue entre deux concepts, Revue Internationale de Psychosociologie, vol. 14, $\mathrm{n}^{\circ} 32$, p. 121-140.

Bhola R., Verheul I., Thurik A.R., Grilo I. (2006), Explaining engagement levels of opportunity and necessity entrepreneurs, JENA economic research paper $\mathrm{n}^{\circ}$ 2007-089. Blackburn R. A., Ram M. (2006), Fix or fixation? The contributions and limitations of entrepreneurship and small firms to combating social exclusion, Entrepreneurship and Regional Development, vol. 18, $\mathrm{n}^{\circ}$ 1, p. 73-89.

Block J., Koellinger P. (2009), I can get no satisfaction : necessity entrepreneurship and procedural utility, Kyklos international review for social sciences, vol. 62, $\mathrm{n}^{\circ} 2$, p.191-209.

Block J., Kohn K., Miller D., Ullrich K. (2014), Necessity Entrepreneurship and Competitive Strategy, research paper $n^{\circ} 8219$, Institute for the Study of Labor (IZA), Bonn.

Block J., Wagner M. (2010), Necessity and Opportunity Entrepreneurs in Germany: Characteristics and Earnings Differentials, Small Business Review, vol. 62, p. 154-174. Borges Da Silva R. (2013), Taxonomie et typologie: est-ce vraiment des synonymes ?, Santé Publique, vol. 25, p. 633-637. 


\section{Auteurs invités: Une approche typologique de l'entrepreneuriat de nécessité}

Christel TESSIER-DARGENT, Alain FAYOLLE

Brasseur M., Coord. (2010), Entrepreneuriat et Insertion, Bruxelles, Bruylant.

Bureau S. (2011), Petite revue sur la revue de littérature à l'usage des candides, Le Libellio d'AEGIS, vol. 7, n 4, p. 65-73.

Caliendo M., Hogenacker J., Künn S., Wießner F. (2015), Subsidized Start-Ups out of unemployment: A Comparison to Regular Business Start-Ups, IZA discussion paper, $\mathrm{n}^{\circ} 8817$.

Carlsrud A., Brännback M. (2011), Entrepreneurial motivations: what do we still need to know?, Journal of Small Business Management, vol. 49 n¹, p. 9-26.

Claret M., Ruane M. (2010), Nene and her business: entrepreneurship by necessity, International Journal of entrepreneurship, vol. 14, p.75-80.

Clark K., Drinkwater S. (2000), Pushed out or pulled in? Self-employment among ethnic minorities in England and Wales, Labour Economics, vol. 7, n 5, p. 603-628.

Couteret P. (2010), Peut-on aider les entrepreneurs contraints? Une étude exploratoire, Revue de l'Entrepreneuriat, vol. 9, $\mathrm{n}^{\circ}$ 2, p. 6-33.

Cowling M., Bygrave W. (2003), Entrepreneurship and Unemployment: Relationship Between Unemployment and Entrepreneurship in 37 Nations Participating in the Global Entrepreneurship Monitor, Babson College.

Fayolle A., Nakara W. (2012), Création par nécessité et précarité : la face cachée de l'entrepreneuriat, Economies et Sociétés, Série «Etudes Critiques en Management », $\mathrm{KC}, \mathrm{n}^{\circ} 2$, p. 1729-1764.

Fraser S. (2009), Is there ethnic discrimination in the UK market for small business, International Small Business Journal, vol. 27, n5, p.583-607.

Gartner W. (1985), A conceptual framework for describing the phenomenon of new venture creation, Academy of Management Review, vol. 10, $\mathrm{n}^{\circ}$ 4, p. 696-706.

Giacomin O., Guyot J.L., Janssen F., Lohest O. (2007), Novice creators: personal identity and push and pull dynamics, conference paper, International Council for Small Business and Entrepreneurship (ICSB) World Conference, At Turku (Finland), vol. 52, p.1-30.

Girard B. (2007), Au début, il y a le salariat..., Revue internationale de psychosociologie, vol. 13, p. 219-233.

Grüner H. (2010), Entrepreneurship in Germany and the role of the new selfemployed, Journal of Business Economics and Management, vol. 7, $\mathrm{n}^{\circ}$ 2, p. 59-67.

Hammarstedt M. (2001), Immigrant self-employment in Sweden - its variation and some possible determinants, Entrepreneurship and Regional Development, vol. 13, p.147-162.

Henrekson M. (2005), Entrepreneurship: a weak link in the welfare state?, Industrial and Corporate Change, vol. 14, $\mathrm{n}^{\circ} 3$, p. 437-467.

Hernandez E.M. (2006), Les trois dimensions de la décision d'entreprendre, Revue française de gestion, vol. 9, p. 337-357.

RIMHE - Revue Interdisciplinaire Management, Homme \& Entreprise

n²2 - Eté 2016

- 90 - 


\section{Auteurs invités : Une approche typologique de l'entrepreneuriat de nécessité Christel TESSIER-DARGENT, Alain FAYOLLE}

Hernandez L., Nunn N., Warnecke T. (2012), Female entrepreneurship in China: opportunity- or necessity-based?, International Journal of Entrepreneurship and Small Business, vol. 15, $\mathrm{n}^{\circ}$ 4, p.411-434.

Kantola J. (2013), Narrating coping experiences of necessity entrepreneurs, thèse de doctorat, Université de Wasa, Finlande.

Kodithuwakku S., Rosa P. (2002), The entrepreneurial process and economic success in a constrained environment, Journal of Business Venturing, vol. 6, n ${ }^{\circ} 17$, p. 431-465. Legrand L., Stervinou S., Lemaitre C. (2012), Vers une meilleure compréhension des bénéficiaires du microcrédit entrepreneurial en France : Proposition d'une typologie des parcours des porteurs de projet, Management international, vol. 16, n², p. 161182.

Levratto N., Serverin E. (2012), L'auto-entrepreneur, instrument de compétitivité ou adoucissant de la rigueur? Bilan de trois années de fonctionnement du régime, Revue de la régulation, vol. 12 - https://regulation.revues.org/9879.

Maâlaoui A., Germon R. (2014), Entretien avec Didier Roche : « Les gens différents développent d'extraordinaires compétences », Entreprendre \& Innover, vol. 1, $\mathrm{n}^{\circ} 20$, p. $78-86$.

Mandják T., Bárdos C., Neuman-Bódi E., Németh S., Simon J. (2011), To solve the impossible: From necessity to success with the help of business network, IMP Journal, vol. 5, $\mathrm{n}^{\circ} 3, \mathrm{p} .212-223$.

Maritz A. (2004), New Zealand necessity entrepreneurs, International Journal of Entrepreneurship and Small Business, vol. 1, n 1, p. 255-263.

Mintzberg H. (1980), Structure in 5's : a synthesis of the research on organization design, Managements Science, vol. 26, $\mathrm{n}^{\circ}$ 3, p. 322-341.

Mitchell D.T., Campbell N.D. (2009), Corruption's Effect on Business Venturing Within the United States, American Journal of Economics and Sociology, vol. 68, $\mathrm{n}^{\circ} 5$, p. 1135-1152.

Nakara W., Fayolle A. (2013), Les «bad» pratiques d'accompagnement à la création d'entreprise. Le cas des entrepreneurs par nécessité, Revue Française de Gestion, vol.38, n $228-229$, p. 231-251.

Niitykangas H., Littunen H., Kinnunen U. (1998), Forced founding - does it differ?, The Finnish Journal of Business Economics, vol. 1, p. 86-104.

Orhan M. (2001), Women Business Owners in France: The Issue of Financing Discrimination, Journal of Small Business Management, vol. 39, p. 95-102.

Reynolds P.D., Camp S.M., Bygrave W.D., Autio E., Hay M. (2001), Global Entrepreneurship Monitor: 2001 Executive Report, Kauffman Center for Entrepreneurial Leadership.

Robichaud Y., Lebrasseur R., Nagarajan K.V. (2010), Necessity and Opportunitydriven Entrepreneurs in Canada: An Investigation into their Characteristics and an

RIMHE - Revue Interdisciplinaire Management, Homme \& Entreprise

n²2 - Eté 2016 


\section{Auteurs invités : Une approche typologique de l'entrepreneuriat de nécessité Christel TESSIER-DARGENT, Alain FAYOLLE}

Appraisal of the Role of Gender, Journal of Applied Business and Economics, vol. 11, $\mathrm{n}^{\circ} 1$, p. 59-88.

Schoar A. (2009), The Divide between Subsistence and Transformational Entrepreneurship, in Lerner J., Stern S. (Eds.), National Bureau of Economic Research Innovation: Policy and the Economy, Chicago, University of Chicago Press, p. 57-81. Schumpeter J.A. (1934), Theory of Economic Development, Harvard University Press. Serviere L. (2010), Forced to entrepreneurship: modeling the factors behind necessity entrepreneurship, Journal of Business and Entrepreneurship, vol. 22, $\mathrm{n}^{\circ}$ 1, p. 37-53. Shane S. (2009), Why encouraging more people to become entrepreneurs is bad public policy, Small Business Economics, vol. 33, p. 141-149.

Shapero A. (1975), The Displaced Uncomfortable Entrepreneur, Psychology Today, vol. 42, p. 83-88.

Singh G., Denoble A. (2003), Early Retirees As the Next Generation of Entrepreneurs, Entrepreneurship Theory and Practice, vol.27, $\mathrm{n}^{\circ}$ 3, p. 207-226.

Smeaton D. (2003), Self-employed workers: calling the shots or hesitant independents? A consideration of the trends, Work Employment and Society, vol. 17, $\mathrm{n}^{\circ} 2$, p. 379-391.

Sternberg R. (2011), Kick-starting Economic Recovery. The Role of Entrepreneurship in the Structural Funds, Conference Federal Ministry of Labour and Social Affairs, Berlin - http://www.cop-ie.eu/sites/default/files/Sternberg_Vortrag_COPIE_31-3-2011.pdf .

Thurik R., Carree M-A., Van Steel A., Audretsch D-B. (2008), Does self-employment reduce unemployment?, Journal of Business Venturing, vol. 23, n 6, p. 673-686.

Tyszka T., Cieslik J. (2011), Motivation, self-efficacy, and risk attitudes among entrepreneurs during transition to a market economy, Journal of Socio-Economics, vol.40, $\mathrm{n}^{\circ} 2$, p. 124-131.

Valéau P. (2006), L'accompagnement des entrepreneurs durant les périodes de doute, Revue de l'Entrepreneuriat, vol. 5, $\mathrm{n}^{\circ}$ 1, p. 31-57.

Van Der Zwan P., Hessels J. (2013), Start-up motivation and (in)voluntary exit, Scales Research Reports, $n^{\circ} 201309$, EIM Business and Policy Research

Vivant E. (2013), Etre auto-entrepreneur, c'est pas si facile. Les ambigüités d'une nouvelle catégorie juridique, Actes journées d'études : Le travail indépendant : santé et conditions de travail.

Wennekers S., Van Stel A., Thurik R., Reynolds P. (2005), Nascent entrepreneurship and the level of economic development, Small Business Economics, vol. 24, $\mathrm{n}^{\circ} 3$, p.293-309.

Wong P.K., Ho Y.P., Autio E. (2005), Entrepreneurship, innovation and economic growth: Evidence from GEM data, Small Business Economics, vol. 24, p. 335-350.

Yaniv E., Brock D. (2012), Reluctant entrepreneurs: Why they do it and how they do it, Ivey Business Journal, pp. 1-8 - http://iveybusinessjournal.com/publication/reluctantentrepreneurs-why-they-do-it-and-how-they-do-it/ .

RIMHE - Revue Interdisciplinaire Management, Homme \& Entreprise

n²2 - Eté 2016

-92 - 